\title{
Carrier transport mechanisms in InSb diffusion $p-n$ junctions
}

\author{
A. Sukach ${ }^{1}$, V. Tetyorkin ${ }^{1}$, A. Voroschenko ${ }^{1}$, A. Tkachuk ${ }^{2}$, M. Kravetskii ${ }^{1}$, I. Lucyshyn ${ }^{1}$ \\ ${ }^{1} V$. Lashkaryov Institute of Semiconductor Physics, NAS of Ukraine, \\ 41, prospect Nauky, 03028 Kyiv, Ukraine \\ ${ }^{2}$ V. Vinnichenko Kirovograd State Pedagogical University, Kirovograd, Ukraine \\ Phone: 38 (044) 525-54-61, e-mail: teterkin@isp.kiev.ua
}

\begin{abstract}
The linearly-graded $p$ - $n$ junctions were prepared by diffusion of cadmium into $n$-InSb(100) substrate with the electron concentration $n \approx 1.6 \cdot 10^{15} \mathrm{~cm}^{-3}$ at the temperature $T=77 \mathrm{~K}$. Passivation and protection of mesa structures have been carried out using thin films of CdTe. Forward and reverse current-voltage characteristics were investigated within the temperature range $77 \ldots 156 \mathrm{~K}$. It has been found that the total dark current consists of generation-recombination and tunneling current components, which are dominant at high $(T=120 \ldots 156 \mathrm{~K})$ and low $(T<120 \mathrm{~K})$ temperatures, respectively. Experimental results have been explained using the model of a nonhomogeneous $p-n$ junction. It has been shown that in the linearly-graded $p$ - $n$ junction with the rather thick $(\sim 1 \mu \mathrm{m})$ depletion region tunneling current flows through the states related to dislocations in the depletion region. The performed estimation of electrical parameters of diffusion InSb $p$-n junctions allows to predict behavior of InSb-based photodiodes at operation temperatures $T>77 \mathrm{~K}$.
\end{abstract}

Keywords: infrared, InSb, linearly-graded junctions, dislocations, tunneling.

Manuscript received 19.01.14; revised version received 11.06.14; accepted for publication 29.10.14; published online 10.11.14.

\section{Introduction}

$\mathrm{InSb}$ photodiodes are widely used for detection of infrared radiation within the spectral range 3 to $5 \mu \mathrm{m}$. High structural perfection (low density of dislocations and low-angle boundaries), uniformity and stability of bulk InSb allow production of focal-plane arrays and matrices [1-3]. Development of $2052 \times 2052 \mathrm{InSb}$ photodiode matrix has been reported in [4]. The photodiodes are usually produced by ion implantation of beryllium, magnesium [5] or thermal diffusion of zinc [2] and cadmium into $n$-InSb bulk substrates $[1,3,4,7]$.

Despite the fact that the development of high-quality InSb photodiodes is known for a long time [5], the problem of improving basic technical parameters and characteristics remains valid until now [7-9]. Especially important is to understand reasons of frequently observed excess dark currents in the photodiodes, which lead to worsening their performances. Availability of excess current in a photodiode means that the measured current can not be explained by bulk diffusion and the generationrecombination carrier transport mechanism. For instance, the excess current associated with the surface leakage was observed at temperatures $77 \ldots 110 \mathrm{~K}$ in the reverse-biased planar InSb $p^{+}-n$ junctions [2]. Similarly, this current was detected in mesa junctions at forward biases [10]. The excess current in InSb photodiodes is ascribed to tunneling $[11,12]$, trap-assisted tunneling [7] and dislocations [13]. Note that in the cited works, except [2], studies were performed at the fixed temperature $77 \mathrm{~K}$. The purpose of this paper is to study carrier transport mechanisms in a wide temperature range. It is clear from the above consideration is important from both scientific and practical point of view. 


\section{Samples and experimental techniques}

InSb $p-n$ junctions were produced by diffusion of cadmium into $n$-InSb monocrystalline substrates with the (100) crystallographic orientation. The electron concentration and mobility at $T=77 \mathrm{~K}$ were $1.6 \cdot 10^{15} \mathrm{~cm}^{-3}$ and $6.4 \cdot 10^{5} \mathrm{~cm}^{2} / \mathrm{V} \cdot \mathrm{s}$, respectively. The surface damaged layer in substrates was removed by chemical dynamic polishing using a polishing etchant $2 \% \mathrm{Br}_{2}-98 \% \mathrm{HBr}$. The quality of the polished surface was controlled by an interference microscope and by measuring its roughness. The average dislocation density determined by selective etching was $\sim 300 \mathrm{~cm}^{-2}$ [14]. Diffusion of $\mathrm{Cd}$ was carried out for $30 \mathrm{~min}$ using the two-temperature method. The substrate and source temperatures were $\sim 400$ and $380{ }^{\circ} \mathrm{C}$, respectively. To prevent re-evaporation of components from the substrate, an additional powder of Sb and In was loaded into ampoule to ensure conditions of vapor saturation. The thickness of the substrate was close to $500 \mu \mathrm{m}$. The depth of $p$ - $n$ junction was determined by measuring the sign of thermo-emf during the layer-by-layer etching of $p$-type doped layer. Mesa structures with the area $A \approx 1.4 \cdot 10^{-2} \mathrm{~cm}^{2}$ and the depth of $p-n$ junction close to $3 \mu \mathrm{m}$ were prepared.

The mirror-like surface of the substrate was observed after $\mathrm{Cd}$ diffusion, but the surface roughness slightly increased from $\sim 0.03$ in the initial substrate up to $\sim 0.05 \mu \mathrm{m}$. The average concentration of holes in the doped surface layer $(7 \pm 1) \cdot 10^{18} \mathrm{~cm}^{-3}$ at the temperature $77 \mathrm{~K}$ was estimated from differential Hall effect measurements [15]. Ohmic contacts were made using thermal vacuum deposition of In- $\mathrm{Zn}$ alloy and In on $p$ and $n$-type region of the substrate, respectively. Passivation and protection of mesa structures was made using deposition of thin $(0.5 \ldots 0.6 \mu \mathrm{m}) \mathrm{CdTe}$ films. This passivation results in the surface states density $(0.5 \ldots 1.0) \cdot 10^{11} \mathrm{eV}^{-1} \mathrm{~cm}^{-2}$ at the $\mathrm{InSb} / \mathrm{CdTe}$ interface, which is 2 to 3 times lower than in the case of anodic oxidation of InSb $[16,17]$. The current-voltage characteristics and high-frequency $(f=1 \mathrm{MHz})$ barrier capacitance were measured for characterization of photodiodes.

\section{Results and discussion}

Typical current-voltage characteristics measured at the temperature $77 \mathrm{~K}$ is shown in Fig. 1. At the bias voltage $U= \pm 250 \mathrm{mV}$, the rectification coefficient was $6 \cdot 10^{3}$. As seen from Fig. 1, the reverse current smoothly increases with the bias increase. The breakdown voltage was in the range $0.7 \ldots 0.8 \mathrm{~V}$, whereas the diffusion potential was $\mathrm{e} U_{D} \approx 160 \mathrm{meV}$. The measured capacitance-voltage characteristics were better linearized in coordinates $C^{-3}-U$ (Fig. 2), indicating formation of a linearlygraded $p-n$ junction. The dopant concentration gradient $a \approx 1.0 \cdot 10^{19} \mathrm{~cm}^{-4}$ as well as the diffusion voltage close to $190 \mathrm{mV}$ were determined from the capacitance measurements. The depletion layer thickness at zero bias $W_{0} \approx 1.2 \mu \mathrm{m}$ was estimated. Experimental data are summarized in Table.

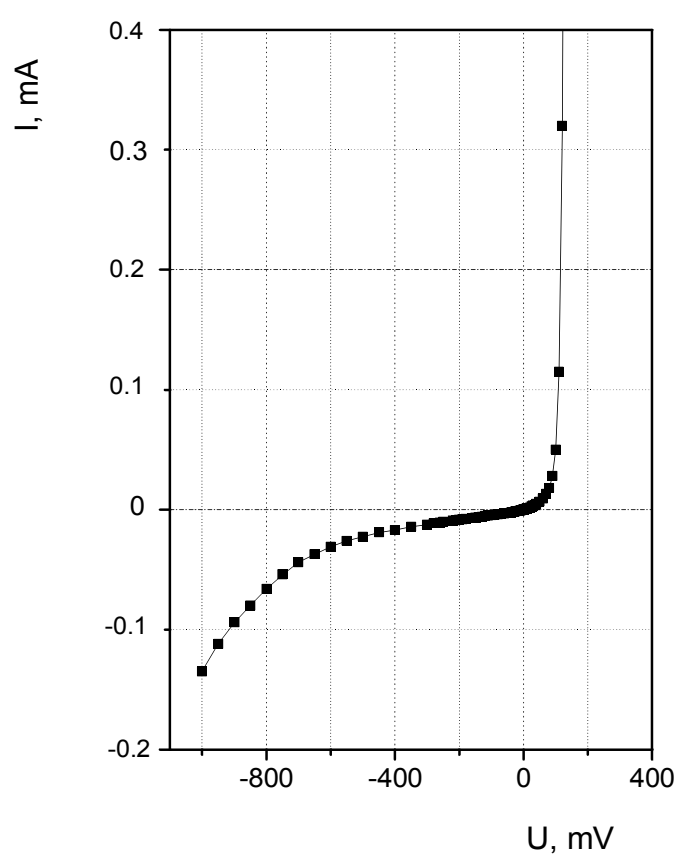

Fig. 1. Current-voltage characteristics of diffusion $\mathrm{InSb} p-n$ junctions at $77 \mathrm{~K}$.

Table. Experimental and calculated parameters of InSb diffusion $p$ - $n$ junctions.

\begin{tabular}{|c|c|c|c|c|c|c|c|}
\hline$T, \mathrm{~K}$ & $I_{02}, \mathrm{~A}$ & $\beta$ & $U_{0}, \mathrm{mV}$ & $n_{i}, \mathrm{~cm}^{-3}$ & $W_{0}, \mu \mathrm{m}$ & $\tau_{0}, \mathrm{~s}$ & $R_{S}, \Omega$ \\
\hline 77 & $4 \cdot 10^{-9}$ & 1.6 & 160 & $1.6 \cdot 10^{9}$ & 1.2 & $1.1 \cdot 10^{-7}$ & 1.5 \\
\hline 116 & $1.6 \cdot 10^{-5}$ & 1.6 & 115 & $1.6 \cdot 10^{12}$ & 1.1 & $2.5 \cdot 10^{-8}$ & 1.7 \\
\hline 134 & $2.6 \cdot 10^{-4}$ & 1.6 & 90 & $1.1 \cdot 10^{13}$ & 1.0 & $9.5 \cdot 10^{-9}$ & 1.9 \\
\hline 156 & $1.3 \cdot 10^{-3}$ & 1.5 & 65 & $6.3 \cdot 10^{13}$ & 0.9 & $9.8 \cdot 10^{-9}$ & 2.1 \\
\hline
\end{tabular}

Notes: $U_{0}$ - current cutoff voltage, $\tau_{0}-$ carrier lifetime in the depletion region 


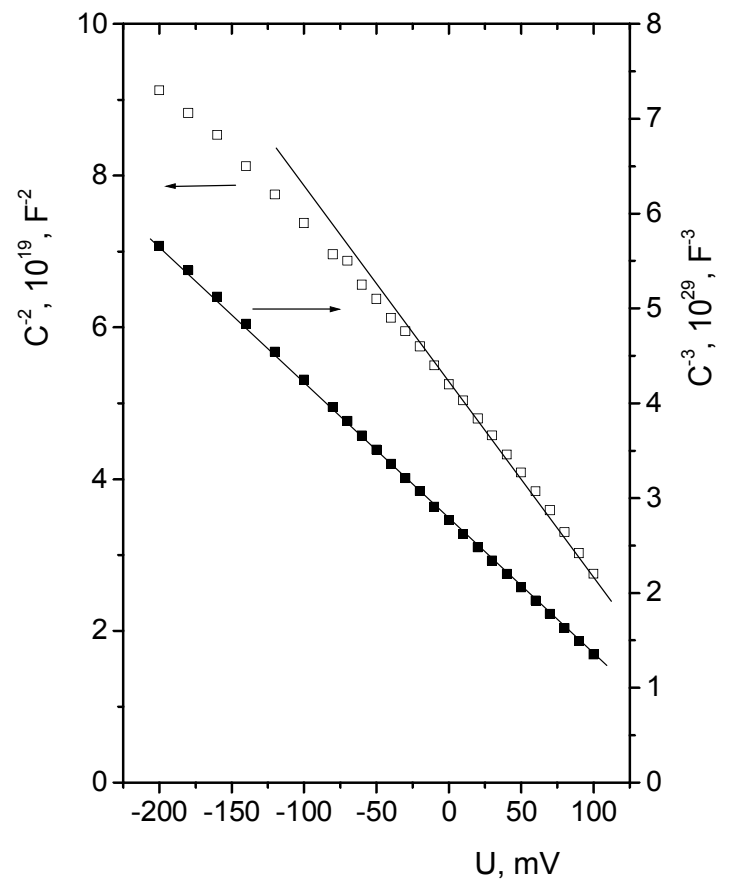

Fig. 2. High-frequency (1 $\mathrm{MHz})$ capacitance-voltage characteristics of diffusion InSb $p-n$ junctions at $77 \mathrm{~K}$ plotted in different coordinates.

The current-voltage characteristics measured at different temperatures are shown in Fig. 3. Note some peculiarities in the behavior of forward $I-U$ characteristics. At $T=77 \mathrm{~K}$, two exponential parts are observed, which can be described by the following equation:

$$
I=I_{01} \exp \left[\frac{e\left(U-I R_{S}\right)}{E_{0}}\right]+I_{02} \exp \left[\frac{e\left(U-I R_{S}\right)}{\beta k T}\right],
$$

where $I_{01}, I_{02}$ are the pre-exponential factors; $E_{0} \approx$ $29 \mathrm{meV}$ is the characteristic energy; $\beta \approx 1.6$ is the ideality coefficient; $R_{S} \approx 1.5 \mathrm{Ohm}$ is the series resistance. At higher temperatures only one exponential part (curve 2) is observed. Deviation from the exponential dependence at bias voltages $U>U_{D}$ is explained by the series resistance effect. Moreover, due to this effect with further increase in temperature $(T>116 \mathrm{~K})$, the exponential part is not observed at all (curve 3 ). The $I-U$ dependence corrected to the series resistance is shown in Fig. 3 (curve 4).

Note that the diode effect is not observed at bias voltages $|U| \leq 10 \mathrm{mV}$ on the forward and reverse currentvoltage characteristics, which is caused by the surface leakage current. At the same time, rather low values of shunt voltages indicate effectiveness of passivation by using CdTe films. Because of investigated junctions are characterized by large ratio of the junction area to the perimeter length, the contribution of the surface leakage at higher biases seems to be low.

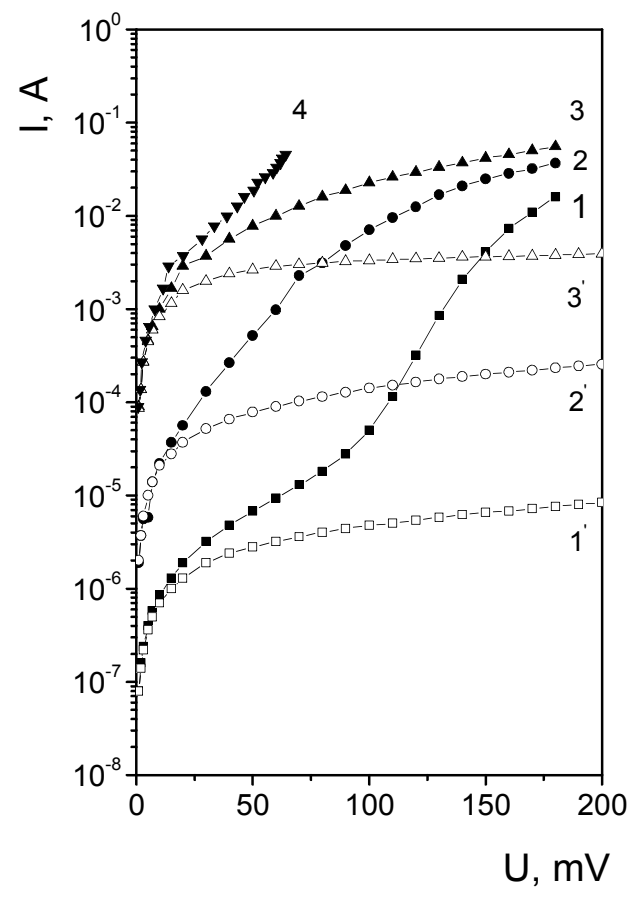

Fig. 3. Forward (closed dots) and reverse (open dots) $I-U$ curves at temperatures, K: $77\left(1,1^{\prime}\right), 116\left(2,2^{\prime}\right), 156\left(3,3^{\prime}\right)$. The curve 4 is corrected to the series resistance effect.

Results of experimental data processing are shown in Table. The intrinsic concentration was calculated using the formulas given in $[18,19]$, where $N_{c}\left(\mathrm{~cm}^{-3}\right)=$ $8.0 \cdot 10^{12} T^{3 / 2}, N_{v}\left(\mathrm{~cm}^{-3}\right)=1.4 \cdot 10^{15} T^{3 / 2}$ are the effective density of states in the conduction and valence band, respectively. The temperature dependence of the band gap $E_{g}$ has been approximated by the formula

$E_{g}(\mathrm{eV})=0.24-6.0 \cdot 10^{-4} T^{2} /(T+500)$.

To understand the behavior of reverse $I-U$ characteristics, experimental results are shown in double logarithmic coordinates (Fig. 4). As seen, they are satisfactorily approximated by a power law $I \sim U^{n}$, where the exponent $n$ is varied with temperature. At $T=$ $77 \mathrm{~K}$ (curve 1 ) in the voltage range $10^{-3}<U \leq 10^{-2}$, the linear dependence is observed with $n \approx 1.0$ followed by the sub-linear one with $n \approx 0.8$ at the bias voltages $2 \cdot 10^{-2}<U \leq 0.2$. Finally, at $U>0.3 \mathrm{~V}$ the current tends to a sharp increase that may be due to the breakdown state of a junction. Similar behavior of the reverse current is also observed at $T=116 \mathrm{~K}$ (curve 2). With temperature increasing (curve 3 ), the sub-linear curve with $n=0.3$ is observed at $U>0.07 \mathrm{~V}$, which is typical for the generation current in a linearly-graded $p-n$ junction [20]. Such behavior of the reverse current is explained by predominant contribution of tunneling current within the temperature range $77 \ldots 116 \mathrm{~K}$, while at $T=156 \mathrm{~K}$ it is mainly determined by the generation carrier component. 


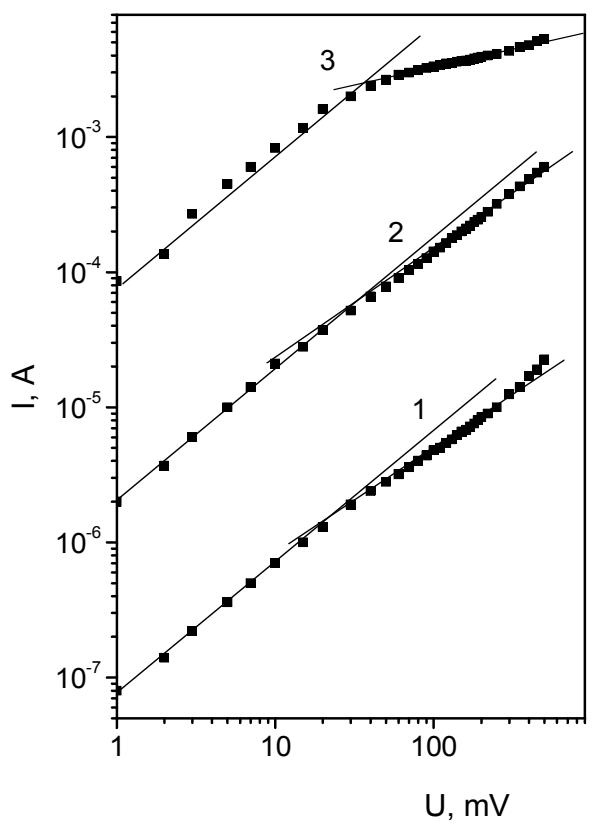

Fig. 4. Reverse current-voltage charateristics at temperatures, K: 77 (1), $116(2), 156(3)$.

The temperature dependence of the resistance-area product $R_{0} A$ at the forward bias voltage of $5 \mathrm{mV}$ is shown in Fig. 5. As seen, it increases with temperature decreasing at temperatures from 160 to $120 \mathrm{~K}$ with the activation energy $\Delta E=0.14 \mathrm{eV}$. With further decrease of temperature $(T<120 \mathrm{~K})$ a tendency to saturation is seen. Thus, the temperature dependence of $R_{0} A$ is explained by dominant contribution of generation and tunnel currents at high and low temperatures, respectively. This conclusion is based on the value of the activation energy, which is typical for the generation current in the depletion region, as well as on the value of the ideality coefficient at $T<120 \mathrm{~K}$ (see Figs 3 and 4). Note that in the absence of tunneling current at the temperature $77 \mathrm{~K}$ the product $R_{0} A$ could be close to $3.5 \cdot 10^{4} \mathrm{Ohm}^{\cdot} \mathrm{cm}^{2}$. This value is almost one order of magnitude higher than that in commercially available InSb photodiodes [21].

Thus, based on experimental results described above, one can conclude that the investigated junctions are linearly-graded. The excess current observed in these junctions has tunneling nature. Since the thickness of the depletion layer $W_{0}$ decreases from $1.2 \mu \mathrm{m}$ at $77 \mathrm{~K}$ to $1.0 \mu \mathrm{m}$ at $134 \mathrm{~K}$ (see Table), the interband tunneling cannot be dominant at rather low bias voltages $|U| \leq \pm U_{D}$. Therefore, it is most likely that tunneling occurs through deep defect states localized in the depletion layer [22-25]. Several models of tunneling current in infrared photodiodes were proposed in the literature [1, 20, 22, 23]. For instance, in the reverse-biased $\mathrm{HgCdTe}$ photodiodes, the soft breakdown was explained by the trap-assisted tunneling mechanism [22, 23]. However, as in the case of InAs $p$ - $n$ junctions [25], investigated InSb junctions were found to be substantially influenced by ultrasonic treatment. So, to explain experimental results at forward and reverse biases, the model of tunneling current through dislocations was chosen [24]. According to this model, the forward tunneling current is approximated by an equation similar to the first component in Eq. (1), and the temperature dependence of the pre-exponential factor is given by the formula $I_{0} \sim \exp (b T)$, where $b=\gamma / E_{0}$ and $E_{0}$ is the characteristic energy.

Figs $6 \mathrm{a}$ and $6 \mathrm{~b}$ show temperature dependences of the dark current in the investigated junction. As seen, in $\lg I-10^{3} / T$ coordinates the current exhibits similar behavior for reverse and forward bias voltages. The activation energy $\Delta E \approx 0.14 \mathrm{eV}$ was found from the slope of the linear parts at temperatures $T>116 \mathrm{~K}$. Approximation of experimental data by the formula $I \sim \exp (b T)$ results in the average value of $b \approx 2.9 \cdot 10^{-2} \mathrm{~K}^{-1}$

The diffusion potential and energy gap as a function of temperature are shown in Fig. 7. The value of $e U_{D}=$ $250 \mathrm{meV}$ determined from the extrapolation to zero temperature is close to the energy gap of $\operatorname{InSb}[18,19]$. From the slope of $e U_{D}$ vs. $T$ dependence values of coefficients $\gamma \approx 1.2 \cdot 10^{-3} \mathrm{eV} / \mathrm{K}$ and $b=\gamma / E_{0} \approx 4.1 \cdot 10^{-2} \mathrm{~K}^{-1}$ were found. As seen, the value of $b$ is close to that found from the temperature dependence of current. At temperatures $T \geq 70 \mathrm{~K}$, the energy gap is approximated by the linear function $E_{\mathrm{g}}(T)=\Delta_{0}-\alpha T$, where $a \approx 2.0 \cdot 10^{-4} \mathrm{eV} / \mathrm{K}$ for the temperature range 77 to $160 \mathrm{~K}$ and $\Delta_{0} \approx 250 \mathrm{meV}$ is close to $E_{g}(0 \mathrm{~K}) \approx 240 \mathrm{meV}$. The linear approximation is necessary to estimate $\gamma[24]$ :

$$
\gamma \approx \alpha+k \ln \left(\frac{N_{c} N_{v}}{n p}\right) .
$$

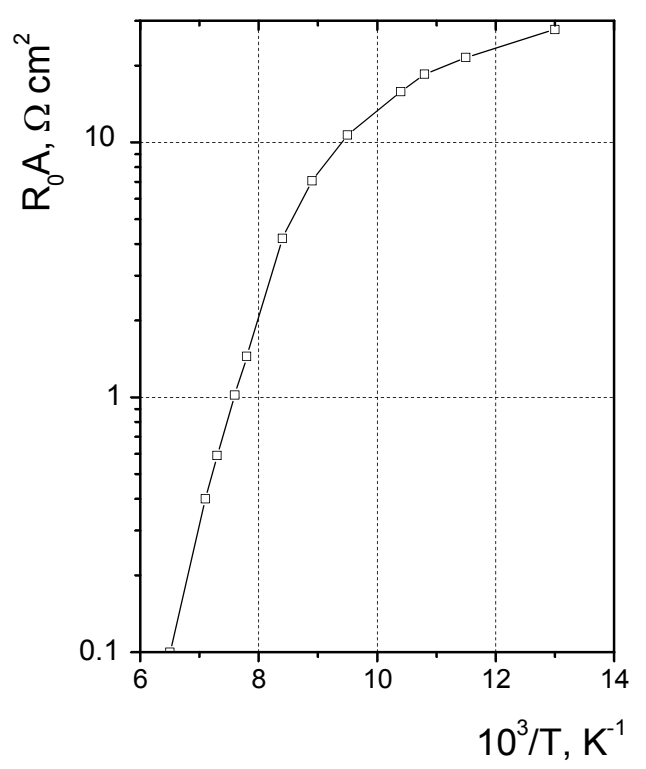

Fig. 5. Temperature dependence of $R_{0} A$ at the bias voltage $5 \mathrm{mV}$. 

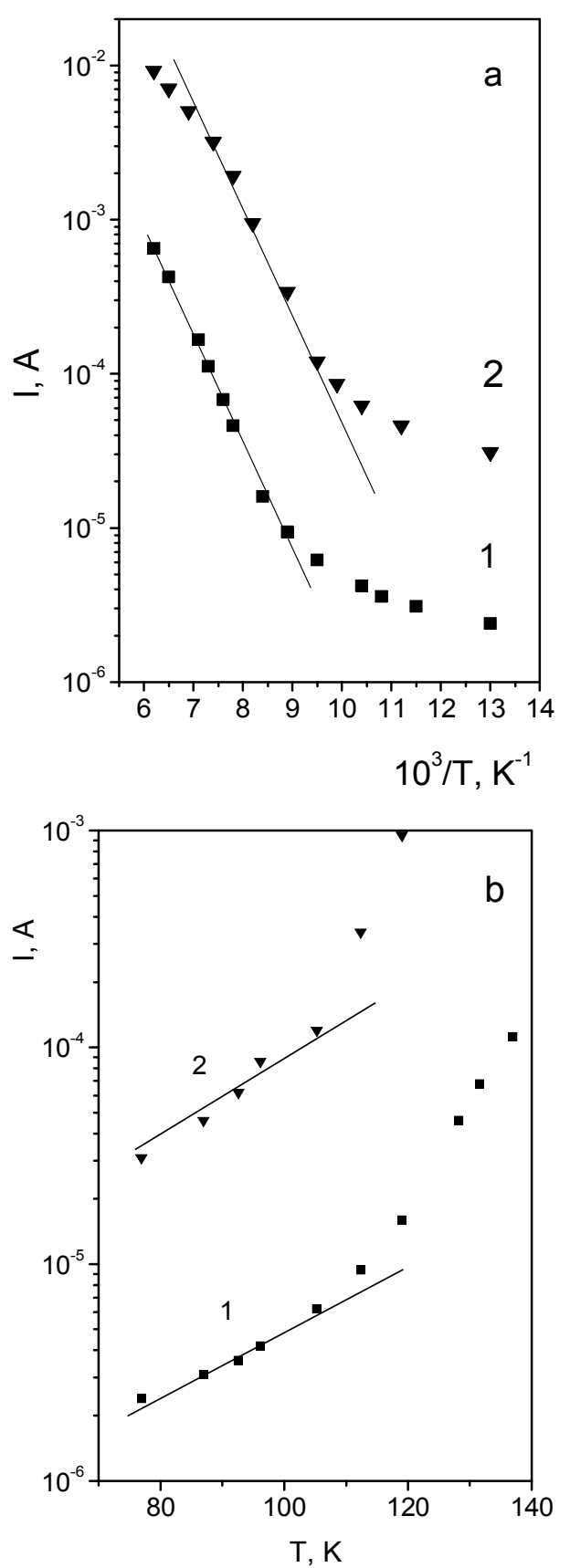

a)

Fig. 6. $(a, b)$ Temperature dependence of the dark current at $5 \mathrm{mV}(1)$ and $-50 \mathrm{mV}(2)$ plotted in different coordinates.

To estimate the coefficient $\gamma$, the concentration of carriers at the edge of the depletion region can be calculated using the formula $n=p=a W_{0} / 2 \approx$ $\approx 6.0 \cdot 10^{14} \mathrm{~cm}^{-3}$ for values of $W_{0}$ given in Table. The calculated and experimental data $\left(1.1 \cdot 10^{-3}\right.$ and $1.2 \cdot 10^{-3} \mathrm{eV} / \mathrm{K}$, respectively) are in good agreement. The model developed in [24] allows us to estimate the dislocation density in the depletion layer

$I_{0}=e \rho v A \exp \left(-\frac{e U_{D}}{E_{0}}\right)$, where $I_{0}$ is the pre-exponential factor; $\rho$ - density of dislocations; $v$ - Debye frequency. At the temperature $77 \mathrm{~K}$, experimental parameters are as follows: $I_{0} / A=$ $9.3 \cdot 10^{-5} \mathrm{~A} / \mathrm{cm}^{2}, e U_{D}=160 \mathrm{meV}, E_{0}=29 \mathrm{meV}$ and $v \approx 3.3 \cdot 10^{12} \mathrm{~s}^{-1}$. The frequency $v$ is estimated using the Debye temperature $T_{\mathrm{D}} \approx 160 \mathrm{~K}$ for $\operatorname{InSb}[18,19]$. The calculated dislocation density $\rho \approx 4.4 \cdot 10^{4} \mathrm{~cm}^{-2}$ is two orders of magnitude higher than that in the starting substrates. Rather high density of dislocations may be caused by relaxation of mechanical stresses that arise during dopant $\mathrm{Cd}$ diffusion into substrates.

In the investigated junctions, tunneling and generation-recombination current flows through different parts of $p-n$ junction, namely: through the junction parts enriched with dislocations and those parts that are free of dislocations, respectively. At low bias voltages $U \geq 3 k T / e$, the tunneling current is approximated by the first term of Eq. (1), whereas the generation current is approximated by the second term at higher bias voltages. At temperatures $T \geq 116 \mathrm{~K}$, the generation current is dominant over the whole range of bias voltages (Fig. 3, curve 2). Tunneling of carriers in the model [24] occurs in the form of successive jumps along the dislocation line initiated by phonons. This model has been successively used for explanation of experimental data in $\mathrm{GaP}$ diode structures that were characterized by a high dislocation density [24]. However, it seems that the dislocation density in the investigated $\mathrm{InSb}$ junctions is overestimated. Also, this model does not consider impurity centers, forming Cottrell atmosphere. The tunneling mechanism that includes participation of Cottrell atmospheres has been considered in application to InAs photodiodes in [25]. Obviously, discrimination of carrier transport mechanisms in the linearly-graded InSb $p$ - $n$ junctions requires additional study, but participation of dislocations in the carrier transport seems to be doubtless.

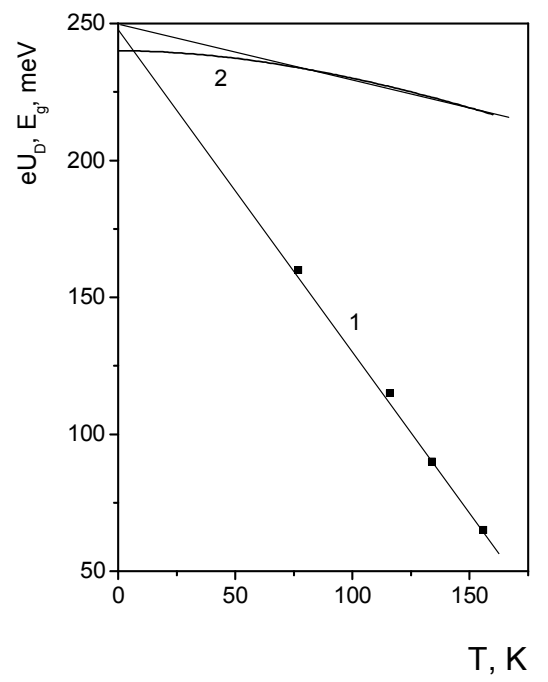

Fig. 7. Temperature dependence of diffusion potential $e U_{D}(1)$ and band gap $E_{g}(2)$. 


\section{Conclusions}

1. The excess current is investigated in InSb $p-n$ junctions prepared by diffusion of $\mathrm{Cd}$ into monocrystalline substrates of $n$-type conductivity. The linear distribution of dopant atoms has been found from the capacitance-voltage measurements. The mesa structure junctions were passivated by $\mathrm{CdTe}$ films.

2. Experimental results have been explained using the model of inhomogeneous $p-n$ junction. It is shown that tunneling of carriers occurs through the states in the gap related to dislocations in the depletion region. The generation-recombination current flows through the depletion region free of dislocations.

\section{References}

1. A. Rogalski, Infrared Photon Detectors. SPIE Optical Eng. Press, N.Y., 1995.

2. P.V. Biryulin, V.I. Turin, E.B. Yakimov, Investigation of characteristics of photodiode arrays based on InSb // Semiconductors, 38(4), p. 498-503 (2004).

3. A.M. Filachev, I.D. Burlakov, A.I. Dirochka et al., High-speed photodetector $128 \times 128$ cells based on $\mathrm{InSb}$ with frame accumulation and range finder function // Prikladnaia Fizika, 2, p. 21-25 (2005), in Russian.

4. A. Rogalski, Optical detectors for focal plane arrays // Optoelectron. Rev. 12(2), p. 221-245 (2004).

5. C.E. Hurwitz, I.P. Donnely, Planar InSbphotodiodes fabricated by $\mathrm{Be}$ and $\mathrm{Mg}$ ion implantation // Solid State Electron. 18(9), p. 753756 (1975).

6. V.P. Astahov, D.A. Gindin, V.V. Karpov, A.V. Talimov, Results of development of InSb photodiodes with ultralow dark current for highsensitivity IR CCD // Prikladnaia Fizika, 3, p. 6871 (2002), in Russian.

7. M. Moradi, M. Daraee, M. Hajian et al., Optimum concentration of InSb photodiode for minimum low reverse bias leakage current // Ukr. J. Phys. 55(4), p. 422-424 (2010).

8. V.P. Astahov, D.A. Gindin, V.V. Karpov et al., On the possibility of increasing the current sensitivity of photodiodes based on InSb // Prikladnaia Fizika, 2, p. 73-79 (1999), in Russian.

9. V.P. Astakhov, D.A. Gindin, V.V. Karpov, AV. Talimov, Thermal stability increase in InSb photodiodes // Prikladnaia Fizika, 1, p. 56-62 (2002), in Russian.
10. V.A. Bogatyrev, A.A. Gavrilov, G.A. Kochurin et al., Electrical and photoelectric properties of p-n transitions on $\mathrm{InSb}$ prepared by introduction of zinc ions with subsequent diffusion distillation // Sov. Phys. Semicond. 12(11), p. 2106-2109 (1978).

11. B.S. Kerner, V.V. Osipov, O.V. Smolin et al., On the mechanism of excess currents in p-n junctions // Sov. Phys. Semicond. 20(9), p. 1739-1742 (1986).

12. V.P. Astahov, V.F. Dudkin, B.S. Kerner et al., Mechanisms of burst noise p-n junctions // Microelectronics, 18(5), p. 455-463 (1989), in Russian.

13. A.V. Sukach, V.V. Tetyorkin, N.M. Krolevec, Tunneling current via dislocations in InAs and InSb infrared photodiodes // Semiconductor Physics, Quantum Electronics and Optoelectronics, 14(4), p. 416-420 (2011).

14. I.F. Dawald, The kinetics and mechanism of formation of anode films on single crystal $\mathrm{InSb} / / J$. Electrochem. Soc. 104(4), p. 244-251 (1957).

15. D.K. Schroder, Semiconductor Material and Device Characterization. Wiley, 2006.

16. I.V. Varlamov, L.A. Vjukov, O.V. Kulikova et al., Photomemory effect in InSb-CdTe heterojunctions // Sov. Phys. Semicond. 15(12), p. 2423-2426 (1981).

17. Y.A. Bykovskii, L.A. Packs, A.G. Dudoladov et al., Investigation of MIS film structures based on CdTe-InSb // Pisma Zhurnal Techn. Fiziki, 9(7), p. 1071-1074 (1983), in Russian.

18. O. Madelung, Semiconductor. Basis Data. Springer, Berlin, 1996.

19. htpp://www.ioffe.ru/SVA/NSM/semicond/.

20. S.M. Sze, Physics of Semiconductor Devices. Wiley, N.Y., 1981.

21. Indium Antimonide Detectors. Catalog. Judson Technologies LLC, 2012.

22. D. Rosenfeld and G. Bahir, A model for the trap assisted tunneling mechanism in diffused $n-p$ and implanted $n^{+}-p$ HgCdTe photodiodes // IEEE Trans. Electron. Devices, 39(7), p. 1638-1645 (1992).

23. Y. Nemirovsky and A. Unicovsky, Tunneling and $1 / f$ noise currents in HgCdTe photodiodes // J. Vac. Sci. Technol. B, 10(4), p. 1602-1610 (1992).

24. V.V. Evstropov, M. Dzhumaeva, Yu.V. Zhilaev et al., Dislocation origin and a model of the excessive tunnel current in $\mathrm{GaP} p-n$ structures // Semiconductors, 34(11), p. 1357-1362 (2000).

25. V. Tetyorkin, A. Sukach, A. Tkachuk, InAs Infrared Photodiodes, In: Advances in Photodiodes, Ed. Gian-Franco Dalla Betta, InTech, 2011. 